\title{
Constrained Point-Set Embeddability of Planar Graphs ${ }^{\star}$
}

\author{
Emilio Di Giacomo ${ }^{1}$, Walter Didimo ${ }^{1}$, Giuseppe Liotta ${ }^{1}$, \\ Henk Meijer ${ }^{2}$, and Stephen Wismath ${ }^{3}$ \\ ${ }^{1}$ Dip. di Ingegneria Elettronica e dell'Informazione, Università degli Studi di Perugia \\ \{digiacomo, didimo, liotta\}@diei.unipg.it \\ ${ }^{2}$ Roosevelt Academy, The Netherlands \\ h.meijer@roac.nl \\ ${ }^{3}$ Department of Mathematics and Computer Science, University of Lethbridge \\ wismath@cs.uleth.ca
}

\begin{abstract}
This paper starts the investigation of a constrained version of the point-set embeddability problem. Let $G=(V, E)$ be a planar graph with $n$ vertices, $G^{\prime}=\left(V^{\prime}, E^{\prime}\right)$ a subgraph of $G$, and $S$ a set of $n$ distinct points in the plane. We study the problem of computing a point-set embedding of $G$ on $S$ subject to the constraint that $G^{\prime}$ is drawn with straight-line edges. Different drawing algorithms are presented that guarantee small curve complexity of the resulting drawing, i.e. a small number of bends per edge. It is proved that: (i) If $G^{\prime}$ is an outerplanar graph and $S$ is any set of points in convex position, a point-set embedding of $G$ on $S$ can be computed such that the edges of $E \backslash E^{\prime}$ have at most 4 bends each. (ii) If $S$ is any set of points in general position and $G^{\prime}$ is a face of $G$ or if it is a simple path, the curve complexity of the edges of $E \backslash E^{\prime}$ is at most 8. (iii) If $S$ is in general position and $G^{\prime}$ is a set of $k$ disjoint paths, the curve complexity of the edges of $E \backslash E^{\prime}$ is $O\left(2^{k}\right)$.
\end{abstract}

\section{Introduction}

The problem of computing a planar drawing of a graph on a given set of points in the plane is a classical subject of investigation both in the graph drawing and in the computational geometry literature. The input is a planar graph $G$ with $n$ vertices and a set of $n$ distinct points in the plane. The output is a drawing of $G$ such that each vertex is mapped to a distinct point of $S$ and no two edges cross each other. Besides the intrinsic theoretical interest of studying the interplay between the topology of the graph and the geometry of the given set of points, the question is in part justified by the variety of graph drawing applications where some or all of the vertices are constrained at fixed locations (see, e.g., [17]).

Different versions of the problem have been investigated. In the point-set embeddability problem with given mapping the function that associates each vertex of $G$ with a distinct point of $S$ is given as part of the input. Halton [10] shows that a planar graph always admits a point-set embedding with given mapping on any set of $n$ points but he

\footnotetext{
* Research partially supported by the MIUR Project "MAINSTREAM: Algorithms for massive information structures and data streams" and by NSERC.
} 
does not give any result about the number of bends per edge. Pach and Wenger [16] describe an algorithm to compute a point-set embedding with given mapping of a planar graph with at most $120 n$ bends per edge; they also prove that $\frac{m}{40^{3}}$ bends per edge may be necessary in some cases ( $m$ is the number of edges of the graph). Both the upper and the lower bounds of Pach and Wenger have been recently reduced by Badent et al. [1] to $3 n+2$ and $\frac{n}{8}$, respectively.

The point-set embeddability problem without mapping allows the drawing algorithm to choose for each vertex $v$ of $G$ the point of $S$ that represents $v$. Algorithms to compute straight-line point-set embedding without mapping of trees [311]15] and outerplanar graphs [2] of graphs admitting a straight-line point-set embedding without mapping on any set of points [9], Kaufmann and Wiese [14] study the point-set embeddability problem with bends. They show that every planar graph admits a point-set embedding without mapping with at most two bends per edge and that this bound is worst-case optimal.

The above two versions of the problem have also been studied in a unifying framework. In a $k$-colored point set embedding each vertex of $G$ and each point of $S$ is given one of $k$ colors and the drawing algorithm can map a vertex of color $i$ to any point of $S$ having the same color. If $k=1$ the problem coincides with the point-set embeddability problem without mapping; if $k=n$ we have the point-set embeddability problem with mapping; if the input specifies a mapping of $n_{1}$ vertices to $n_{1}$ points while there is no mapping for the remaining $n-n_{1}$, we have a $k$-colored point-set embeddability problem with $k=n_{1}+1$. A limited list of papers about the $k$-colored point set embeddability includes [1|4|5|8|11|12 13$]$.

This paper studies a natural extension of the point-set embeddability problem without mapping. It is assumed that a subgraph of $G$ is given whose edges are required to be drawn as straight-line segments. Our input is a graph $G=(V, E)$ with $n$ vertices, a set $S$ of $n$ distinct points in the plane, and a subgraph $G^{\prime}=\left(V^{\prime}, E^{\prime}\right)$ of $G$. We want to compute a point-set embedding of $G$ with small curve complexity (i.e. a small number of bends per edge) and such that $G^{\prime}$ is drawn with straight-line edges. It may be worth recalling that a recent paper [6] has proved that if $G$ is a tree, $G^{\prime}$ is a tree with $k$ edges, and a mapping from the vertices of $G^{\prime}$ to a subset of the points of $S$ is given, then a point-set embedding where the edges of $G^{\prime}$ are straight-line can have $O(k)$ curve complexity. A fundamental difference between the setting of [6] and this paper is that here we do not assume that the mapping from the subgraph to the point set is given as part of the input. A high level description of the results in the paper is as follows.

- We prove that if $S$ is a set of points in convex position, then $G$ admits a point-set embedding on $S$ with the edges of $G^{\prime}$ drawn straight-line if and only if $G$ has a planar embedding $\psi$ such that the embedding of $G^{\prime}$ induced by $\psi$ is outerplanar and each vertex of $V \backslash V^{\prime}$ is on the external face of $G^{\prime}$ in $\psi$. Furthermore we show that, when the drawing exists, it can be computed in such a way that the edges of $E \backslash E^{\prime}$ have at most 4 bends each.

- We extend the above investigation to sets of points in general position. If either $G^{\prime}$ is a face of $G$ or if it is a simple path, a point-set embedding of $G$ on any set of points in general position exists such that the edges of $G^{\prime}$ are straight-line edges and the edges of $E \backslash E^{\prime}$ have at most 8 bends each. 
- We finally consider the situation where $G^{\prime}$ is a set of $k$ disjoint paths and $S$ is any set of points in general position. In this case a point-set embedding exists where the edges of $G^{\prime}$ are straight-line edges and the edges of $E \backslash E^{\prime}$ have $O\left(2^{k}\right)$ bends each.

In the remainder of the paper some proofs are omitted for reasons of space.

\section{Preliminaries}

Let $G=(V, E)$ be a graph. A drawing $\Gamma$ of $G$ maps each vertex $v$ of $G$ to a distinct point $p(v)$ of the plane and each edge $e=(u, v)$ of $G$ to a simple Jordan curve connecting $p(u)$ and $p(v)$. Drawing $\Gamma$ is planar if no two distinct edges intersect except at common end-vertices. Graph $G$ is planar if it admits a planar drawing. A planar drawing $\Gamma$ of $G$ partitions the plane into topologically connected regions called the faces defined by $\Gamma$. The unbounded face is called the external face. The boundary of a face is its delimiting cycle described by the circular list of its edges and vertices. A face is simple if its boundary is a simple cycle.

An embedding of a planar graph $G$ is an equivalence class of planar drawings that define the same set of faces, that is, the same set of face boundaries. A planar graph $G$ together with the description of a set of faces $F$ is called an embedded planar graph. A maximal embedded planar graph is such that all faces are triangles, that is, the boundary of each face has three vertices and three edges. Given any embedded planar graph $G$, it is easy to add edges that split the faces of $G$ in order to obtain a maximal embedded planar graph that includes $G$. A graph $G$ is outerplanar if it admits an embedding such that all vertices of $G$ belong to a common face, which we can always choose as the external face.

A subdivision of a graph $G$ is a graph obtained from $G$ by replacing each edge by a path with at least one edge. Internal vertices on such a path are called division vertices, the edges of such path are called sub-edges.

Let $G=(V, E)$ be a planar graph with $n$ vertices and let $S$ be a set of $n$ points in the plane. We say that the points of $S$ are in general position if no three points of $S$ lie on the same line. A point-set embedding of $G$ on $S$, denoted as $\Gamma(G, S)$, is a planar drawing of $G$ such that each vertex is mapped to a distinct point of $S . \Gamma(G, S)$ is called a geometric point-set embedding if all edges are drawn straight-line. Let $G^{\prime}=\left(V^{\prime}, E^{\prime}\right)$ be a subgraph of $G$. A point-set embedding of $G$ on $S$ with straight-line subgraph $G^{\prime}$, denoted as $\Gamma\left(G, G^{\prime}, S\right)$, is a point-set embedding of $G$ on $S$ such that the edges of $G^{\prime}$ are drawn as straight-line segments. The edges of $G$ can be partitioned, with respect to $G^{\prime}$, in the following four sets:

Blue Edges. $E_{\text {blue }}=E^{\prime}$;

Red Edges. $E_{\text {red }}=\left\{(u, v) \in E \backslash E^{\prime} \mid u \notin V^{\prime} \wedge v \notin V^{\prime}\right\}$;

Black Edges. $E_{\text {black }}=\left\{(u, v) \in E \backslash E^{\prime} \mid u \in V^{\prime} \wedge v \notin V^{\prime}\right\}$;

Green Edges. $E_{\text {green }}=\left\{(u, v) \in E \backslash E^{\prime} \mid u \in V^{\prime} \wedge v \in V^{\prime}\right\}$.

Let $G=(V, E)$ be a planar graph. A 2-page book embedding of $G$ is a planar drawing of $G$ such that all vertices of $G$ are represented as points of a horizontal line $\ell$ called the spine and each edge is drawn in one of the two half-planes defined by $\ell$. The 
half-plane above $\ell$ is called the top page, while the half-plane below the spine is called the bottom page. An edge of a 2-page book embedding is completely contained either in the top page or in the bottom page. Let $e_{1}=\left(u_{1}, v_{1}\right)$ and $e_{2}=\left(u_{2}, v_{2}\right)$ be two edges in the same page of a 2-page book embedding. Assume, without loss of generality, that $u_{1}$ is to the left of $v_{1}$ on $\ell$, that $u_{2}$ is to the left of $v_{2}$ on $\ell$, and that $u_{1}$ is to the left of $u_{2}$ on $\ell$. A crossing between $e_{1}$ and $e_{2}$ can be avoided only if $u_{1}, u_{2}, v_{1}$, and $v_{2}$ do not appear in this order along the spine. On the other hand, if $u_{1}, u_{2}, v_{1}$, and $v_{2}$ do not appear in this order along the spine the two edges can easily be drawn without crossing (for example they can be drawn as circular arcs). Thus, the fact that two edges in a same page cross or not depends only on the relative position of their endvertices.

A monotone topological book embedding of a planar graph $G$ is a 2-page book embedding of a subdivision of $G$ such that each edge $(u, v)$ of $G$ has at most one division vertex $d$ and: (i) $u, d$, and $v$ appear in this order along the spine; (ii) sub-edge $(u, d)$ is in the bottom page; (iii) sub-edge $(d, v)$ is in the top page.

In [7] an algorithm to compute a monotone topological book embedding of a planar graph was presented. The next lemma, that will be used as a basic tool for the algorithms presented in the next sections, can be proved by exploiting the algorithm of [7] for computing a monotone topological book embedding.

Lemma 1. Let $G=(V, E)$ be an embedded planar graph. If $G$ contains a cycle $C=$ $v_{1}, v_{2}, \ldots, v_{h}$ whose interior is triangulated with edges $\left(v_{1}, v_{i}\right)$ for $3 \leq i \leq h-1$, then there exists a monotone topological book embedding $\gamma$ such that the linear order of the vertices of $C$ in $\gamma$ is $v_{1}, v_{h}, v_{h-1}, \ldots, v_{2}$; also, all edges of $C$ and all edges $\left(v_{1}, v_{i}\right)$ $(3 \leq i \leq h-1)$ are in the bottom page of $\gamma$.

\section{Points in Convex Position}

Let $G$ be a planar graph and let $G^{\prime}$ be a subgraph of $G$. In this section we give a necessary and sufficient condition on the embedding of $G$ and $G^{\prime}$ that guarantees that $G$ has a point-set embedding on any set of points in convex position with straight-line subgraph $G^{\prime}$. We start by giving some technical lemmas.

Lemma 2. Let $G=(V, E)$ be an embedded simply connected outerplanar graph. There exists a graph $G_{a}=\left(V_{a}, E_{a}\right)$ such that: (i) $V_{a}=V$ and $E \subset E_{a}$; (ii) $G_{a}$ is biconnected and outerplanar; (iii) every edge in $E_{a} \backslash E$ is on the external face.

Lemma 3. Let $S$ be a set of points in convex position. It is possible to connect any two points $p$ and $q$ of $S$ with a 1-bend polyline that does not intersect the interior of the convex hull of $S$.

Lemma 4. Let $G=(V, E)$ be a planar graph, let $S$ be any set of points in convex position and let $G^{\prime}=\left(V^{\prime}, E^{\prime}\right)$ be a biconnected subgraph of $G$. Let $G$ admit a planar embedding $\psi$ such that: (i) the embedding of $G^{\prime}$ induced by $\psi$ is outerplanar; and (ii) all vertices of $V \backslash V^{\prime}$ are on the external face of $G^{\prime}$ in the embedding $\psi$. Then $G$ admits a point-set embedding on $S$ with straight-line subgraph $G^{\prime}$. 
Proof. Refer to Fig. 1 for an illustration. Since $G^{\prime}$ is biconnected, the boundary of its external face is a cycle $C$. Maintaining the embedding $\psi$ we change the external face in such a way that an edge $(u, v)$ of $C$ is on the external face. Since the embedding of $G^{\prime}$ induced by $\psi$ is outerplanar, then no vertex of $G^{\prime}$ is inside $C$; also no vertex of $V \backslash V^{\prime}$ is inside $C$. Thus, $C$ contains only blue edges. Let $v_{1}=u, v_{2}=v, \ldots, v_{h}$ be the vertices of $C$ according to their circular ordering. Remove all blue edges inside $C$ and replace them with blue edges of the type $\left(v_{1}, v_{i}\right)$ for $3 \leq i \leq h-1$. We denote the graph obtained from this transformation as $\bar{G}$ and its subgraph consisting of $C$ and of the blue edges inside it as $\overline{G^{\prime}}$. By Lemma 1, the graph $\bar{G}$ admits a monotone topological book embedding $\bar{\gamma}$ such that the linear order of the vertices of $C$ in $\bar{\gamma}$ is $v_{1}, v_{h}, v_{h-1}, \ldots, v_{2}$ and all the edges of $C$ along with the blue edges inside it are in the bottom page of $\bar{\gamma}$.

Let $u_{1}, u_{2}, \ldots, u_{n^{\prime}}$ be the linear ordering of the (real and division) vertices of $\bar{G}$ in $\bar{\gamma}$. We enrich the set $S$ with extra points so that the resulting set $\bar{S}$ has $n^{\prime}$ points in convex position. Let $p_{1}, p_{2}, \ldots, p_{n^{\prime}}$ be the points of $\bar{S}$ according to their clockwise circular ordering. The points of $\bar{S} \backslash S$ are positioned in such a way that $p_{i}$ is an extra point if and only if $u_{i}$ is a division vertex $\left(1 \leq i \leq n^{\prime}\right)$. Vertex $u_{i}$ is mapped to $p_{i}(1 \leq i \leq n)$. All the subedges of $\bar{G}$ that are in the bottom page of $\bar{\gamma}$ will be drawn inside the convex hull $C H(\bar{S})$ of $\bar{S}$ as straight-line segments between their end-vertices. The subedges of $\bar{G}$ that are in the top page of $\bar{\gamma}$ are drawn outside $C H(\bar{S})$ with one bend. More precisely, let $E_{t}$ be the set of sub-edges in the top page of $\bar{\gamma}$. We start drawing an edge $e_{1} \in E_{t}$ such that, when drawn with the technique of Lemma 3 , it leaves the points of $\bar{S}$ representing the end-vertices of the edges of $E_{t}$ not yet drawn, on the convex hull of $\bar{S} \cup\{q\}$, where $q$ is the bend point of $e_{1}$. We now can apply the same drawing technique to the set of subedges $E_{t} \backslash\left\{e_{1}\right\}$ and to the set of points $\bar{S} \cup q$.

Let $\bar{\Gamma}$ be the drawing obtained after replacing the division vertices with bends. We prove now that $\bar{\Gamma}$ is a point-set embedding of $\bar{G}$ on $S$ with straight-line subgraph $\overline{G^{\prime}}$. By construction, each vertex of $\bar{G}$ is mapped to a point of $S$. Let $e_{1}=\left(u_{1}, v_{1}\right)$ and $e_{2}=\left(u_{2}, v_{2}\right)$ be two sub-edges of $\bar{G}$; we prove that $e_{1}$ and $e_{2}$ do not cross. If they are in different pages in $\bar{\gamma}$ then one of them is inside $C H(\bar{S})$ and the other one is outside $C H(\bar{S})$ and therefore they do not cross. Suppose they are both in the bottom page; since they do not cross in $\bar{\gamma}$ then the order of their end-vertices in $\bar{\gamma}$ is either $u_{1}, v_{1}, u_{2}, v_{2}$ or $u_{1}, u_{2}, v_{2}, v_{1}$. Since the circular ordering of the points representing $u_{1}, v_{1}, u_{2}, v_{2}$ is coherent with the linear ordering that these vertices have in $\bar{\gamma}$, then $e_{1}$ and $e_{2}$ do not cross. If $e_{1}$ and $e_{2}$ are both in the top page, then they are drawn with one bend. Without loss of generality assume that $e_{1}$ is drawn before $e_{2}$. By construction, the polyline representing $e_{2}$ does not intersect the interior of the convex hull of $\bar{S} \cup$ $\left\{q_{i} \mid q_{i}\right.$ is the bend point of an edge drawn before $\left.e_{2}\right\}$ and therefore it does not cross $e_{1}$. By Lemma 1 all edges of $\overline{G^{\prime}}$ are in the bottom page of $\bar{\gamma}$ and therefore they are drawn straight-line.

We prove now that the drawing $\Gamma$ obtained from $\bar{\Gamma}$ by restoring the original blue edges inside $C$ is a point-set embedding of $G$ on $S$ with straight-line subgraph $G^{\prime}$. Since $C$ is drawn straight-line and the points are in convex position, then $C$ is drawn as a convex polygon $P$. Also, by Lemma 1 the linear order of the vertices $v_{1}, v_{2}, \ldots, v_{h}$ of $C$ in $\bar{\gamma}$ is $v_{1}, v_{h}, v_{h-1}, \ldots, v_{2}$; it follows that their circular order on the boundary of 


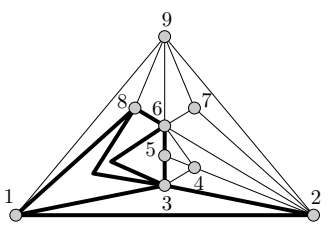

(a)

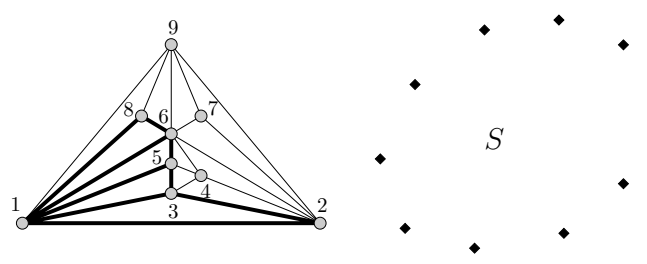

(b) (c)

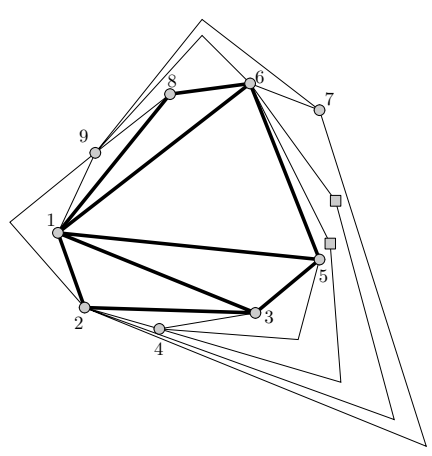

(d)

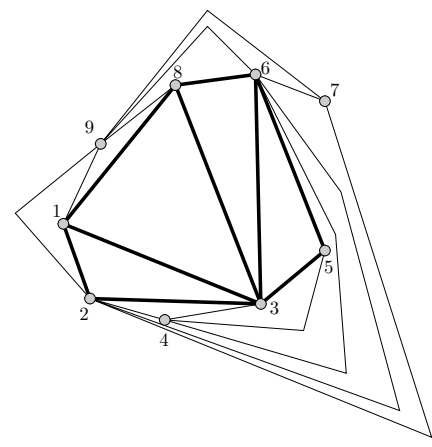

(e)

Fig. 1. (a) A maximal embedded planar graph $G$; the bold edges highlight a biconnected outerplanar subgraph $G^{\prime}$. (b) The subgraph $G^{\prime}$ is transformed into a new subgraph $\overline{G^{\prime}}$ with all chords incident on vertex 1. (c) A set $S$ of 9 points in convex position. (d) A point-set embedding of $\bar{G}$ on $\bar{S}$ with straight-line subgraph $\overline{G^{\prime}} ; \bar{S}$ is a set of points obtained from $S$ by adding two extra points. (d) A point-set embedding of $G$ on $S$ with straight-line subgraph $G^{\prime}$.

$P$ is $v_{1}, v_{2}, \ldots, v_{h}$, i.e. the same ordering they have in the embedding of $\psi$. Hence the original blue edges can be restored without creating any crossing.

Theorem 1. Let $G=(V, E)$ be a planar graph, let $S$ be any set of points in convex position and let $G^{\prime}=\left(V^{\prime}, E^{\prime}\right)$ be a connected subgraph of $G$. $G$ admits a point-set embedding $\Gamma\left(G, G^{\prime}, S\right)$ on $S$ with straight-line subgraph $G^{\prime}$ if and only if $G$ admits a planar embedding $\psi$ such that: (i) the embedding of $G^{\prime}$ induced by $\psi$ is outerplanar; (ii) all vertices of $V \backslash V^{\prime}$ are on the external face of $G^{\prime}$ in the embedding $\psi$.

Also, if $\Gamma\left(G, G^{\prime}, S\right)$ exists, it can be computed so that: If $G^{\prime}$ is biconnected, then every edge not in $G^{\prime}$ has at most 2 bends per edge; If $G^{\prime}$ is simply connected, then every edge not in $G^{\prime}$ has at most 4 bends per edge.

Proof. Let $\Gamma\left(G, G^{\prime}, S\right)$ be a point-set embedding of $G$ on $S$ with straight-line subgraph $G^{\prime}$. Consider the drawing $\Gamma^{\prime}$ of $G^{\prime}$ in $\Gamma\left(G, G^{\prime}, S\right)$. Since the points of $S$ are in convex position and $\Gamma^{\prime}$ is a straight-line drawing, all vertices of $G^{\prime}$ are on the external face of $\Gamma^{\prime}$ and therefore $G^{\prime}$ is outerplanar. Also, all points distinct from those representing the vertices of $V^{\prime}$ are on the external face of $\Gamma^{\prime}$. It follows that the embedding defined by $\Gamma\left(G, G^{\prime}, S\right)$ is the desired embedding $\psi$. 
Assume now that $G$ does admit an embedding $\psi$ that satisfies the statement; we prove that $G$ admits a point-set embedding on $S$ with straight-line subgraph $G^{\prime}$. If $G^{\prime}$ is biconnected, then this is true by Lemma 4. If $G^{\prime}$ is not biconnected we apply the biconnection procedure described in Lemma 2 to make it biconnected. The dummy edges that are added to make $G^{\prime}$ biconnected can cross black and green edges. Every crossing between a dummy edge and a black/green edge is replaced with a division vertex that splits both the dummy edge and the black/green edge. Each black edge can be split at most once. Namely, let $e=(u, v)$ be a black edge and assume that $u \in V^{\prime}$ and $v \in V \backslash V^{\prime}$. If a dummy edge $e^{\prime}$ crosses $e$, then $u$ is encountered between the two end-vertices of $e^{\prime}$ when walking clockwise along the external boundary of $G^{\prime}$; we say that $e^{\prime}$ covers $u$. If $e$ was crossed more than once then there would be at least two dummy edges that cover $u$. In this case, however, one of the two dummy edges cannot be on the external face of $G^{\prime}$, which is impossible by Lemma 2 Each green edge $e=(u, v)$ can be split at most twice. Namely, with an analogous argument as in the case of black edges, we have that there is at most one edge that covers $u$ and at most one edge that covers $v$.

All the dummy (sub-)edges are considered to be blue edges. Let $e=(u, v)$ be a black edge split by a division vertex $d$ and assume that $u \in V^{\prime}$ and $v \in V \backslash V^{\prime}$ sub-edge $(u, d)$ is considered a blue edge, while sub-edge $(d, v)$ is considered a black edge. Analogously, if $e$ is a green edge split by two division vertices $d_{1}$ and $d_{2}$, the sub-edges $\left(u, d_{1}\right)$ and $\left(d_{2}, v\right)$ are considered to be blue edges, while sub-edge $\left(d_{1}, d_{2}\right)$ is considered to be a green edge. The graph obtained after this transformation will be denoted as $\hat{G}$. This graph has as a subgraph the graph $\hat{G}^{\prime}$ that consists of $G^{\prime}$ plus the blue edges added as dummy edges or obtained by splitting a black/green edge of $G$. $\hat{G}^{\prime}$ is outerplanar and biconnected and by Lemma $2 \hat{G}$ admits a point-set embedding on a suitably augmented set of points $\hat{S}$ with straight-line subgraph $\hat{G}^{\prime}$. Since no edge of $G^{\prime}$ is split with a division vertex, removing dummy edges and replacing division vertices with bends we obtain a point-set embedding of $G$ on $S$ with straight-line subgraph $G^{\prime}$.

We count now the number of bends along red, black, and green edges. If $G^{\prime}$ is biconnected, then each red, black, or green edge $e$ is split by at most one division vertex in the monotone book embedding $\bar{\gamma}$. The two sub-edges obtained from $e$ are in different pages and thus one of them is drawn straight-line and the other one is drawn with one bend. Since the division vertex that splits $e$ is replaced at the end by an additional bend, then the number of bends along $e$ is two.

Suppose now that $G^{\prime}$ is not biconnected and let $e$ be a black edge. Edge $e$ can be split into a blue sub-edge $e_{1}$ and a black sub-edge $e_{2}$ during the biconnection procedure. The drawing of the transformed graph $\hat{G}$ is computed like in the case when $G^{\prime}$ is biconnected, and therefore $e_{1}$ has no bends while $e_{2}$ has at most two bends. Since the division vertex that splits $e$ into $e_{1}$ and $e_{2}$ is replaced by an additional bend, the total number of bends along $e$ is at most three. Let $e^{\prime}$ be a green edge; edge $e$ can be split into a blue sub-edge $e_{1}$, a green sub-edge $e_{2}$, and a blue sub-edge $e_{3}$ during the biconnection procedure. The drawing of the transformed graph $\hat{G}$ is computed like in the case when $G^{\prime}$ is biconnected, and therefore $e_{1}$ and $e_{3}$ have no bends while $e_{2}$ has at most two bends. Since the division vertices that split $e$ into $e_{1}, e_{2}$, and $e_{3}$ are replaced by two additional bends, the total number of bends along $e$ is at most four. 


\section{Points in General Position}

In this section we consider points in general position and consider planar graphs whose subgraph is either a cycle or a path or a set of disjoint paths. Before giving the main results of this section we need to give some additional definitions and to prove a pair of lemmas that will be used subsequently.

Let $\Gamma$ be a drawing of a graph $G$ and let $v$ be a vertex of $G$; we say that $v$ is visible from below in $\Gamma$ if $\Gamma$ does not intersect the open vertical halfline below $v$. Analogously, $v$ is visible from above in $\Gamma$ if $\Gamma$ does not intersect the open vertical halfline above $v$. Throughout this section we assume that the points of $S$ have distinct $x$-coordinate; if this is not the case we can achieve this condition by a suitable rotation of the plane.

Lemma 5. Let $C$ be a cycle and let $S$ be a set of points in general position. $C$ admits a geometric point-set embedding $\Gamma$ on $S$ such that each vertex of $C$ is visible in $\Gamma$ either from above or from below.

Proof. Let $C H(S)$ be the convex hull of $S$ and let $p_{l}$ and $p_{r}$ be the leftmost and the rightmost point of $C H(S)$, respectively. In $C H(S)$ there are two paths from $p_{l}$ to $p_{r}$ : the first one, that we call upper hull, has no point of $S$ above, the other one, that we call lower hull, has no point of $S$ below. Let $p_{l}=p_{1}, p_{2}, \ldots, p_{h}=p_{r}$ be the points in the lower hull ordered from left to right. Let $q_{1}, q_{2}, \ldots, q_{k}$ be the remaining points ordered from right to left.

Let $v_{1}, v_{2}, \ldots, v_{n}$ be the vertices of $C$ ordered clockwise. Notice that $n=h+k$. Vertex $v_{i}$, for $1 \leq i \leq h$, is mapped to point $p_{i}$; vertex $v_{h+i}$, for $1 \leq i \leq k$, is mapped to point $q_{i}$. Cycle $C$ can be divided into two paths with end-vertices in common: $\pi_{1}=$ $v_{1}, v_{2}, \ldots, v_{k}$ and $\pi_{2}=v_{k}, v_{k+1}, v_{k+2}, \ldots, v_{k+m}, v_{1}$. Both $\pi_{1}$ and $\pi_{2}$ are represented by a $x$-monotone polyline and therefore neither the drawing of $\pi_{1}$ nor the drawing of $\pi_{2}$ has a crossing. On the other hand, all points of $\pi_{2}$ are above those of $\pi_{1}$ except for the end-vertices that are in common. Hence there is no crossing between edges of $\pi_{1}$ and edges of $\pi_{2}$. Due to the monotonicity of $\pi_{1}$ and $\pi_{2}$, the vertices of $\pi_{1}$ are visible from below, and those of $\pi_{2}$ are visible from above.

Lemma 6. Let $G=(V, E)$ be an embedded planar graph and let $S$ be a set of points in general position. If $G$ contains a cycle $C=v_{1}, v_{2}, \ldots, v_{h}$ whose interior is triangulated with edges $\left(v_{1}, v_{i}\right)$ for $3 \leq i \leq h-1$, then there exists a point-set embedding of $G$ on $S$ such that: (i) each edge has at most two bends; and (ii) after removing the edges of $C$ and the edges $\left(v_{1}, v_{i}\right)(3 \leq i \leq h-1)$ all vertices of $C$ are visible from below.

Proof. Let $\gamma$ be a monotone topological book embedding of $G$ computed according to Lemma 1 Let $w_{1}, w_{2}, \ldots, w_{n^{\prime}}$ be the real and division vertices of $G$ in the order they appear along the spine of $\gamma$. We enrich the set $S$ with extra points so that the resulting set $S^{\prime}$ has $n^{\prime}$ points and, denoted as $p_{1}, p_{2}, \ldots, p_{n^{\prime}}$ the points of $S^{\prime}$ according to their order along the $x$-direction, $p_{i}$ is an extra point if and only if $w_{i}$ is a division vertex $\left(1 \leq i \leq n^{\prime}\right)$.

We compute a point-set embedding of $G$ on $S^{\prime}$ with the following technique introduced by Kaufmann and Wiese [14]. Vertex $w_{i}$ is mapped to point $p_{i}\left(1 \leq i \leq n^{\prime}\right)$; the edges between vertices that are consecutive along the spine of $\gamma$ (and therefore 
drawn on consecutive points of $S^{\prime}$ ) are drawn straight-line. Let $\sigma$ be a value greater than the maximum slope of a segment $\overline{p_{i} p_{i+1}}\left(1 \leq i \leq n^{\prime}-1\right)$; the remaining edges are drawn as polylines with two segments (and hence one bend) with slope $\sigma$ and $-\sigma$. Using segments with slope $\sigma$ and $-\sigma$ it is possible to draw an edge either above or below the polyline $\pi=\overline{p_{1} p_{2}}, \overline{p_{2} p_{3}}, \ldots, \overline{p_{n^{\prime}-1} p_{n^{\prime}}}$. If a sub-edge $e$ is in the top page of $\gamma$, then it is drawn above $\pi$; if $e$ is in the bottom page of $\gamma$, then it is drawn below $\pi$. The resulting drawing is planar except that 1-bend edges that are incident on the same vertex may contain overlapping segments. To eliminate these overlaps, we perturb the overlapping edges by decreasing the absolute value of their segment slopes by slightly different amounts. The slope changes are chosen to be small enough to avoid creating edge crossings while preserving the planar embedding. See [14] for details.

Via this technique, each sub-edge of $G$ has at most one bend. Since $\gamma$ is a monotone topological book embedding, each edge of $G$ is split into at most two sub-edges. Let $e=(u, v)$ be an edge of $G$ subdivided into two sub-edges $e_{1}$ and $e_{2}$ by a division vertex $d(u, v)$. One of the two sub-edges is in the top page in $\gamma$ and the other one is in the bottom page in $\gamma$. Thus, the point $p$ representing $d(u, v)$ has two segments incident on it: one from above and the other one from below; also, these two segments have the same slope. Since these two segments are the only two incident on $d(u, v)$ no rotation is performed to remove overlaps and therefore, when $d(u, v)$ is removed, no extra bend is created at $p$. It follows that each edge of $G$ has at most two bends.

By Lemma 1, all edges of $C$ and all edges inside $C$ are in the bottom page. Since point-set embedding of $G$ on $S$ preserves the embedding of $G$, then there is no vertex or edge inside the drawing of $C$. Thus, if all edges of $C$ and all edges inside $C$ are removed, the vertices of $C$ are visible from below.

Theorem 2. Let $G$ be an embedded planar graph, let $S$ be any set of points in general position and let $C$ be the boundary of a simple face of $G$. G admits a point-set embedding on $S$ with straight-line subgraph $C$ such that every edge not in $C$ has at most 8 bends per edge.

Proof. We describe how to compute a point-set embedding of $G$ on $S$ with straight-line $C$. Maintaining the embedding of $G$ we change the external face so that some edge $e=(u, v)$ of $C$ is on the external face. Let $v_{1}=u, v_{2}=v, \ldots, v_{h}$ be the vertices of $C$ according to their circular ordering. We add blue edges $\left(v_{1}, v_{i}\right)(3 \leq i<h)$ inside $C$.

Let $S^{\prime} \subseteq S$ be the set containing the first $h$ points along the $x$-direction. A pointset embedding $\Gamma^{\prime}$ of $C$ on $S^{\prime}$ is computed as described in the proof of Lemma 5. The drawing $\Gamma^{\prime}$ is computed so that the circular clockwise order of the vertices is preserved and vertex $v_{1}$ is placed at the leftmost point of $S$.

Let $\gamma$ be a monotone topological book embedding of $G$ computed according to Lemma 1 Let $w_{1}, w_{2}, \ldots, w_{n^{\prime \prime}}$ be the real and division vertices of $G$ in the order they appear along the spine of $\gamma$. We enrich the set $S \backslash S^{\prime}$ with extra points so that the resulting set $S^{\prime \prime}$ has $n^{\prime \prime}$ points and, denoted as $p_{1}, p_{2}, \ldots, p_{n^{\prime \prime}}$ the points of $S^{\prime \prime}$ according to their order along the $x$-direction, $p_{i}$ is an extra point if and only if $w_{i}$ is a division vertex or a vertex of $C\left(1 \leq i \leq n^{\prime \prime}\right)$. We compute a point-set embedding of $G$ on $S^{\prime \prime}$ according to Lemma 6 After this point-set embedding is computed we remove the edges of $C$ and the blue edges inside $C$. Denote as $\Gamma^{\prime \prime}$ the resulting drawing. 


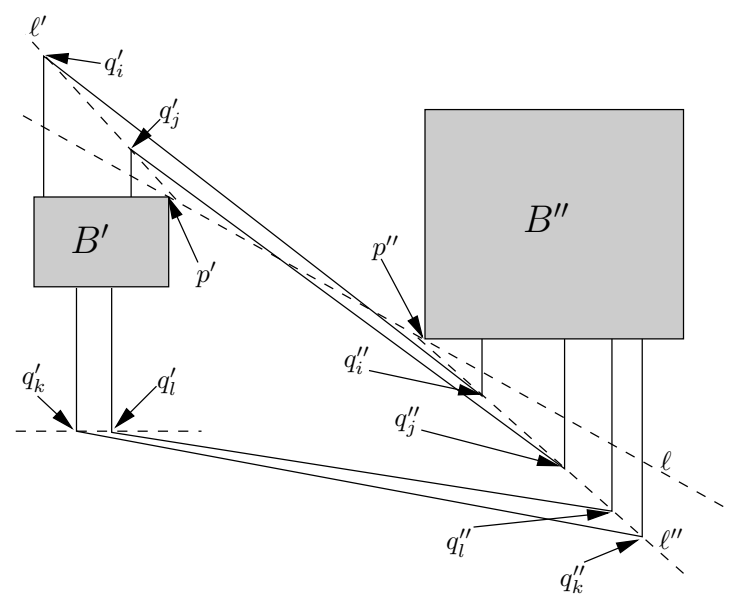

Fig. 2. An illustration for the proof of Theorem 2

Let $v_{i}(1 \leq i \leq h)$ be a vertex of $C$. Let $p_{i}^{\prime}$ be the point representing $v_{i}$ in $\Gamma^{\prime}$, and let $p_{i}^{\prime \prime}$ be the point representing $v_{i}$ in $\Gamma^{\prime \prime}$. Notice that $p_{i}^{\prime}$ is a point of $S$, while $p_{i}^{\prime \prime}$ is not a point of $S$; therefore $v_{i}$ will be represented by $p_{i}^{\prime}$ in the final drawing. All black/green edges incident to $v_{i}$ are incident on point $p_{i}^{\prime \prime}$. We add a polyline from $p_{i}^{\prime}$ to $p_{i}^{\prime \prime}$, in order to make these edges incident to $p_{i}^{\prime}$.

Let $B^{\prime}$ be the bounding box of $\Gamma^{\prime}$ and let $B^{\prime \prime}$ be the bounding box of $\Gamma^{\prime \prime}$. Let $p^{\prime}$ be the top-right corner of $B^{\prime}$ and let $p^{\prime \prime}$ be the bottom-left corner of $B^{\prime \prime}$. Refer to Fig. 2 for an illustration. Let $\ell$ be the straight line through $p^{\prime}$ and $p^{\prime \prime}$. Let $\ell^{\prime}$ be a half-line that is completely above $\ell$ and intersects all vertical lines that intersect $B^{\prime}$; let $\ell^{\prime \prime}$ be a half-line that is completely below $\ell$ and intersects all vertical lines that intersect $B^{\prime \prime}$. Finally, let $\ell_{H}$ be a horizontal line below $B^{\prime}$.

Let $q_{i}^{\prime}$ be the projection of point $p_{i}^{\prime}$ either on line $\ell^{\prime}$, if $p_{i}^{\prime}$ is visible from above, or on line $\ell_{H}$, if $p_{i}^{\prime}$ is visible from below. Notice that the leftmost and rightmost points of $\Gamma^{\prime}$ are visible both from above and from below. We project these two vertices on $\ell^{\prime}$. Let $q_{i}^{\prime \prime}$ be the projection of $p_{i}^{\prime \prime}$ on $\ell^{\prime \prime}$. The polyline connecting $p_{i}^{\prime}$ to $p_{i}^{\prime \prime}$ is $\pi_{i}=\overline{p_{i}^{\prime} q_{i}^{\prime}}, \overline{q_{i}^{\prime} q_{i}^{\prime \prime}}, \overline{q_{i}^{\prime \prime} p_{i}^{\prime \prime}}$.

Notice that, if there are $k>1$ black/green edges incident to a vertex $v_{i}$, then all these edges are represented with a portion in common: the polyline $\pi_{i}$. It is possible to separate these edges by replacing each of the three points $q_{i}^{\prime}, q_{i}^{\prime \prime}, p_{i}^{\prime}$ with a set of $k$ points coherently ordered.

Since both $\Gamma^{\prime}$ and $\Gamma^{\prime \prime}$ are planar, planarity can be proved by showing that the polylines $\pi_{i}(1 \leq i \leq h)$ cross neither $\Gamma^{\prime}$ nor $\Gamma^{\prime \prime}$ and do not cross each other.

Consider a polyline $\pi_{i}(1 \leq i \leq h)$ and assume first that $q_{i}^{\prime}$ is above $B^{\prime}$. Segment $\overline{p_{i}^{\prime} q_{i}^{\prime}}$ does not cross $\Gamma^{\prime}$ because $p_{i}^{\prime}$ is visible from above and it does not cross $\Gamma^{\prime \prime}$ because it is completely to the left of $B^{\prime \prime}$. Segment $\overline{q_{i}^{\prime \prime} p_{i}^{\prime \prime}}$ does not cross $\Gamma^{\prime \prime}$ because $p_{i}^{\prime \prime}$ is visible from below and does not cross $\Gamma^{\prime}$ because it is completely to the right of $B^{\prime}$. Since point $q_{i}^{\prime}$ is above $\ell$ and point $q_{i}^{\prime \prime}$ is below $\ell$, segment $\overline{q_{i}^{\prime} q_{i}^{\prime \prime}}$ crosses $\ell$ between $p^{\prime}$ and $p^{\prime \prime}$ and therefore it crosses neither $\Gamma^{\prime}$ nor $\Gamma^{\prime \prime}$. Suppose now that $q_{i}^{\prime}$ is below $B^{\prime}$. In this case segment $\overline{p_{i}^{\prime} q_{i}^{\prime}}$ does not cross $\Gamma^{\prime}$ because $p_{i}^{\prime}$ is visible from below and it does not cross 
$\Gamma^{\prime \prime}$ because it is completely to the left of $B^{\prime \prime}$. Segment $\overline{q_{i}^{\prime \prime} p_{i}^{\prime \prime}}$ does not cross $\Gamma^{\prime \prime}$ because $p_{i}^{\prime \prime}$ is visible from below and does not cross $\Gamma^{\prime}$ because it is completely to the right of $B^{\prime}$. Point $q_{i}^{\prime}$ is below $B^{\prime}$ by construction; point $q_{i}^{\prime \prime}$ is below $\ell$ and therefore below $B^{\prime \prime}$. It follows that segment $\overline{q_{i}^{\prime} q_{i}^{\prime \prime}}$ crosses neither $\Gamma^{\prime}$ nor $\Gamma^{\prime \prime}$.

Consider now two polylines $\pi_{i}$ and $\pi_{j}(1 \leq i, j \leq h)$. Assume first that $q_{i}^{\prime}$ and $q_{j}^{\prime}$ are both above $B^{\prime}$. Since the drawing $\Gamma^{\prime}$ preserves the clockwise circular order of $C$ and since $v_{1}$ is represented by the leftmost point of $\Gamma^{\prime}$, the order of the projections on $\ell^{\prime}$ is $q_{1}^{\prime}, q_{h}^{\prime}, q_{h-1}^{\prime}, \ldots, q_{h-k}^{\prime}(k \geq 1)$. This means that the order of $q_{i}^{\prime}$ and $q_{j}^{\prime}$ on $\ell^{\prime}$ is the same as the order of $q_{i}^{\prime \prime}$ and $q_{j}^{\prime \prime}$ on $\ell^{\prime \prime}$ and therefore $\pi_{i}$ and $\pi_{j}$ do not cross each other. Suppose now that $q_{i}^{\prime}$ and $q_{j}^{\prime}$ are both below $B^{\prime}$. Since the drawing $\Gamma^{\prime}$ preserves the clockwise circular order of $C$ and since $v_{1}$ is represented by the leftmost point of $\Gamma^{\prime}$, the order of the projections on $\ell_{H}$ is $q_{2}^{\prime}, q_{3}^{\prime}, \ldots, q_{h-k-1}^{\prime}(k \geq 1)$. This means that the order of $q_{i}^{\prime}$ and $q_{j}^{\prime}$ on $\ell_{H}$ is opposite to the order of $q_{i}^{\prime \prime}$ and $q_{j}^{\prime \prime}$ on $\ell^{\prime \prime}$ and therefore $\pi_{i}$ and $\pi_{j}$ do not cross each other. Finally, suppose that $q_{i}^{\prime}$ is above $B^{\prime}$ and $q_{j}^{\prime}$ is below $B^{\prime}$. In this case $q_{j}^{\prime \prime}$ is below and to the right of $q_{i}^{\prime \prime}$, and therefore a crossing is not possible.

We count now the number of bends per edge. Clearly the blue edges are drawn with zero bends in $\Gamma^{\prime}$. The red edges are drawn with two bends in $\Gamma^{\prime \prime}$. Consider a black edge $e$. Edge $e$ is first drawn with two bends in $\Gamma^{\prime \prime}$ with one end-vertex at a point $p_{i}^{\prime \prime}$ $(1 \leq i \leq h)$; the drawing of this edge is then modified with the addition of the polyline $\pi_{i}$. Since polyline $\pi_{i}$ has two bends and an extra bend can exist at point $p_{i}^{\prime \prime}$, then the total number of bends on $e$ is at most 5 . Let $e^{\prime}$ be a green edge. The two end-vertices of $e^{\prime}$ are vertices of $C$ but $e^{\prime}$ is not an edge of $C$. Thus edge $e^{\prime}$ is first drawn with two bends in $\Gamma^{\prime \prime}$ with one end-vertex at a point $p_{i}^{\prime \prime}$ and the other one at a point $p_{j}^{\prime \prime}(1 \leq i, j \leq h)$; the drawing of this edge is then modified with the addition of the two polylines $\pi_{i}$ and $\pi_{j}$. Each of these polylines has 2 bends and two extra bends can exist at $p_{i}^{\prime \prime}$ and $p_{j}^{\prime \prime}$. It follows that $e^{\prime}$ has at most 8 bends per edge.

With techniques similar to that of Theorem 2 the following theorems can be proved.

Theorem 3. Let $G$ be an embedded planar graph, let $S$ be any set of points in general position and let $P$ be a subgraph of $G$ that is a simple path. $G$ admits a point-set embedding on $S$ with straight-line subgraph $P$ such that every edge not in $C$ has at most 8 bends per edge.

Theorem 4. Let $G$ be an embedded planar graph, let $S$ be any set of points in general position and let $P_{1}, P_{2}, \ldots, P_{k}$ be a set of disjoint subgraphs of $G$ each being a simple path. $G$ admits a point-set embedding on $S$ with straight-line subgraph $\bigcup_{i=1}^{k} P_{i}$ such that every edge not in $\bigcup_{i=1}^{k} P_{i}$ has $O\left(2^{k}\right)$ bends per edge.

\section{Open Problems}

1. Theorem 1 shows that for every set of points in convex position and for every outerplanar subgraph a point-set embedding can be computed such that the subgraph has straight-line edges and the remaining edges of the graph have at most four bends each. How hard is it to compute a point-set embedding with the minimum number of bends in total? 
2. Extend Theorems 2 and 3 by considering the case that $G^{\prime}$ is either a tree or a general outerplanar graph. Note that the outerplanar graphs are the largest family of graphs that admit a straight-line point-set embedding without mapping on any set of points [9].

3. We have studied constrained point-set embeddability on any given set of points. Given a planar graph $G$ with $n$ vertices, a non outerplanar subgraph $G^{\prime}$ of $G$, and a set $S$ of $n$ distinct points in convex position, how hard is it to test whether $G$ admits a point-set embedding on $S$ such that $G^{\prime}$ is drawn with straight-line edges and the remaining edges have constant curve complexity?

\section{References}

1. Badent, M., Di Giacomo, E., Liotta, G.: Drawing colored graphs on colored points. Theoret. Comput. Sci. 408(2-3), 129-142 (2008)

2. Bose, P.: On embedding an outer-planar graph on a point set. Comput. Geom. Theory Appl. 23, 303-312 (2002)

3. Bose, P., McAllister, M., Snoeyink, J.: Optimal algorithms to embed trees in a point set. J. Graph Algorithms Appl. 2(1), 1-15 (1997)

4. Di Giacomo, E., Didimo, W., Liotta, G., Meijer, H., Trotta, F., Wismath, S.K.: $k$-colored point-set embeddability of outerplanar graphs. J. Graph Algorithms Appl. 11(1), 29-49 (2008)

5. Di Giacomo, E., Liotta, G., Trotta, F.: On embedding a graph on two sets of points. Internat. J. Found. Comput. Sci. 17(5), 1071-1094 (2006)

6. Di Giacomo, E., Didimo, W., Liotta, G., Meijer, H., Wismath, S.K.: Point-set embeddings of trees with edge constraints. In: Hong, S.-H., Nishizeki, T., Quan, W. (eds.) GD 2007. LNCS, vol. 4875, pp. 113-124. Springer, Heidelberg (2008)

7. Di Giacomo, E., Didimo, W., Liotta, G., Wismath, S.K.: Curve-constrained drawings of planar graphs. Comput. Geom. Theory Appl. 30(1), 1-23 (2005)

8. Di Giacomo, E., Liotta, G., Trotta, F.: Drawing colored graphs with constrained vertex positions and few bends per edge. In: Hong, S.-H., Nishizeki, T., Quan, W. (eds.) GD 2007. LNCS, vol. 4875, pp. 315-326. Springer, Heidelberg (2008)

9. Gritzmann, P., Mohar, B., Pach, J., Pollack, R.: Embedding a planar triangulation with vertices at specified points. Amer. Math. Monthly 98(2), 165-166 (1991)

10. Halton, J.H.: On the thickness of graphs of given degree. Inform. Sci. 54, 219-238 (1991)

11. Ikebe, Y., Perles, M., Tamura, A., Tokunaga, S.: The rooted tree embedding problem into points in the plane. Discrete Comput. Geom. 11, 51-63 (1994)

12. Kaneko, A., Kano, M.: Straight line embeddings of rooted star forests in the plane. Discrete Appl. Math. 101, 167-175 (2000)

13. Kaneko, A., Kano, M.: Semi-balanced partitions of two sets of points and embeddings of rooted forests. Internat. J. Comput. Geom. Appl. 15(3), 229-238 (2005)

14. Kaufmann, M., Wiese, R.: Embedding vertices at points: Few bends suffice for planar graphs. J. Graph Algorithms Appl. 6(1), 115-129 (2002)

15. Pach, J., Törőcsik, J.: Layout of rooted trees. DIMACS Series in Discrete Math. and Theoretical Comput. Sci. 9, 131-137 (1993)

16. Pach, J., Wenger, R.: Embedding planar graphs at fixed vertex locations. Graphs and Combinatorics 17, 717-728 (2001)

17. Sugiyama, K.: Graph Drawing and Applications for Software and Knowledge Engineers. World Scientific, Singapore (2002) 